\title{
GAPDH Pseudogenes and the Quantification of Feline Genomic DNA Equivalents
}

\section{A. Katrin Helfer-Hungerbuehler, Stefan Widmer, and Regina Hofmann-Lehmann}

Clinical Laboratory, Vetsuisse Faculty, University of Zurich, Winterthurerstrasse 260, 8057 Zurich, Switzerland

Correspondence should be addressed to A. Katrin Helfer-Hungerbuehler; khungerbuehler@vetclinics.uzh.ch

Received 17 January 2013; Revised 21 March 2013; Accepted 28 March 2013

Academic Editor: Emanuel Strehler

Copyright (C) 2013 A. Katrin Helfer-Hungerbuehler et al. This is an open access article distributed under the Creative Commons Attribution License, which permits unrestricted use, distribution, and reproduction in any medium, provided the original work is properly cited.

\begin{abstract}
Quantitative real-time PCR (qPCR) is broadly used to detect and quantify nucleic acid targets. In order to determine cell copy number and genome equivalents, a suitable reference gene that is present in a defined number in the genome is needed, preferably as a single copy gene. For most organisms, a variable number of glyceraldehyde-3-phosphate dehydrogenase (GAPDH) pseudogenes have been reported. However, it has been suggested that a single-copy of the GAPDH pseudogene is present in the feline genome and that a GAPDH assay can therefore be used to quantify feline genomic DNA (gDNA). The aim of this study was to determine whether one or more GAPDH pseudogenes are present in the feline genome and to provide a suitable alternative qPCR system for the quantification of feline cell copy number and genome equivalents. Bioinformatics and sequencing results revealed that not just one but several closely related GAPDH-like sequences were present in the cat genome. We thus identified, developed, optimized, and validated an alternative reference gene assay using feline albumin (fALB). Our data emphasize the need for an alternative reference gene, apart from the GAPDH pseudogene, for the normalization of gDNA levels. We recommend using the fALB qPCR assay for future studies.
\end{abstract}

\section{Introduction}

Fluorescence-based quantitative real-time PCR (qPCR) is a highly sensitive method for the detection and quantification of nucleic acids. Due to its conceptual simplicity, sensitivity, specificity, and speed, qPCR applications can be found in a variety of fields, including medicine and the life sciences $[1,2]$. In clinical diagnostics, qPCR is broadly used for the detection and quantification of bacterial and viral loads, gene dosage determination, cancer diagnostics, and applications in forensic medicine [3-7].

To assess the cell number present in a PCR reaction, the coanalysis of suitable reference genes is crucial. Such reference genes should be single-copy number genes and should not frequently undergo genetic alterations, to allow the accurate normalization of genomic DNA (gDNA) samples. In addition, internal control genes are also used to investigate abnormalities in gene number, and amplified oncogenes have been shown to have diagnostic, prognostic, and therapeutic relevance. Thus, TaqMan PCR-based gene quantification assays are also used to identify allelic imbalances (germ-line deletions or amplifications), for example, in individuals suffering from breast cancer, cutaneous melanoma, or nervous system tumors $[8,9]$.

Glyceraldehyde-3-phosphate dehydrogenase (GAPDH) represents a universally expressed reference gene that has many biological roles in addition to its function in glycolysis. A GAPDH assay has been developed for the accurate normalization of feline messenger RNA (mRNA) expression [10], and this assay was recently validated and compared to other potential feline mRNA reference gene assays [11]. Subsequently, the GAPDH assay was also applied as quality control to test for the integrity of the gDNA and the absence of PCR inhibitors $[10,12,13]$. One report suggested that a single copy of the GAPDH pseudogene is present in the feline genome and that the feline GAPDH assay can therefore be used to quantify cell number in feline samples [14]. However, no information on the exact position or the sequence of this GAPDH pseudogene was provided [14]. In contrast, a variable number of GAPDH pseudogenes has been reported for other organisms $[15,16]$. 
Pseudogenes for many different genes have been found in all animal genomes studied so far [17]. Due to the high sequence similarity of the pseudogenes to their "parent" gene, pseudogenes often interfere with PCR or hybridization experiments that are intended to detect the genes only $[18,19]$. Specifically, processed pseudogenes typically lack introns and are therefore well known to hamper data interpretation in mRNA transcription analysis [18, 20]. Promising clinical applications of RT-PCR assays for the purpose of early diagnosis and relapse monitoring of micrometastatic tumor cells have suffered from false-positive results due to their interference with corresponding pseudogenes in the past [19]. Thus, the aim of this study was to (i) investigate the number and sequence(s) of the GAPDH pseudogene(s) in the feline genome, with a specific focus on the GAPDH assay region that is frequently used to normalize genome equivalents [14] and (ii) provide a suitable alternative qPCR system for the normalization of the amount of input gDNA and the determination of cell number in feline samples.

\section{Materials and Methods}

2.1. Sample Description. All cats included in this study were in experimental studies officially approved by the Veterinary Office of the Swiss Canton of Zurich (TVB 30/2003, 59/2005, and 99/2007). The cats were kept in groups under optimal ethological conditions in a barrier facility, as previously described [21]. All cats were euthanized for reasons unrelated to the present study.

Tissue samples were collected upon necropsy from two feline leukemia virus-infected cats (cat B8, female 3.8 years old, and cat 15, neutered male, 1.3 years old). The cats underwent histopathological examination, and samples from the rectum, colon, spleen, and jejunum were collected. Tissues for histology were processed as described [22], histologically examined, and verified to be free of pathological abnormalities. The samples for molecular analysis were snap frozen in liquid nitrogen following collection and stored at $-80^{\circ} \mathrm{C}$ until nucleic acid extraction.

2.2. Nucleic Acid Extractions. Tissues (approximately $25 \mathrm{mg}$ ) were homogenized prior to extraction in $180 \mu \mathrm{L}$ ATL buffer (Qiagen, Hombrechtikon, Switzerland) for genomic DNA isolation. The samples were processed using the DNeasy Blood \& Tissue Kit (Qiagen) following the manufacturer's recommendations. For all nucleic acid extractions, negative controls consisting of $100 \mu \mathrm{L}$ of phosphate-buffered saline were prepared with each batch to monitor cross-contamination.

2.3. GAPDH Pseudogene Sequencing. For the analysis of the GAPDH-like sequences of the domestic cat, gDNA was obtained from tissue samples from two cats (cat B8, rectum and colon, and cat 15 , spleen and jejunum).

Primers were designed using Primer Express software (Version 3, Applied Biosystems, Rotkreuz, Switzerland) based on the GAPDH mRNA sequence [23] to encompass the previously published TaqMan sequence [10]. PCR amplification of the GAPDH pseudogenes using the forward primer binding the exon 2 (GCGCCTGGTCACCAGGGCTGC; pb position: 39-59) and the reverse primer located on the exon 4 (GACTCCACAACATACTCAGCACCAGCATCAC; bp position: 287-257) resulted in an approximately $249 \mathrm{bp}$ fragment. To ensure a high fidelity of amplification, the PCR was performed using Phusion polymerase and HF buffer (Finnzymes, Ipswich, UK), $500 \mathrm{nM}$ of each primer, and $2 \mu \mathrm{L}$ of extracted gDNA in a final volume of $20 \mu \mathrm{L}$. The cycling conditions were as follows: initial denaturation for $2 \mathrm{~min}$ at $98^{\circ} \mathrm{C}$, followed by 40 cycles of $20 \mathrm{sec}$ at $98^{\circ} \mathrm{C}$, $30 \mathrm{sec}$ at $70^{\circ} \mathrm{C}$, and $20 \mathrm{sec}$ at $72^{\circ} \mathrm{C}$ prior to a final elongation at $72^{\circ} \mathrm{C}$ for $10 \mathrm{~min}$. The PCR products were separated by $2.5 \%$ agarose gel electrophoresis, excised from the gel, purified using the GenElute Gel Extraction Kit (Sigma-Aldrich, Buchs, Switzerland), and cloned into the pCRII-TOPO TA cloning vector (Invitrogen, Basel, Switzerland) according to the manufacturer's instructions. A total of 15 of the obtained clones were sequenced by Microsynth (Balgach, Switzerland).

The sequences were analyzed using Clone Manager Professional software version 7.01 (Scientific \& Educational Software, Cary, NC, USA).

2.4. Alternative qPCR Assay Design. In other species, albu$\min (\mathrm{ALB})$ is known to be a single-copy gene and therefore it has been used as an internal control gene $[8,9,24,25]$. In order to determine whether feline ALB (fALB) is also present as a single-copy gene, sequence and gene organization information of the feline genome were retrieved from GenBank (no. NM_001009961) and Ensembl (ENSFCAG00000011854). Subsequently, a fALB qPCR assay was designed. The TaqMan hydrolysis probe and primers for the fALB assay were designed using Primer Express software (Version 2, Applied Biosystems; Table 1). The primer pair (Microsynth, Table 1) was tested to ensure that it amplified a product of the appropriate length using $5 \mu \mathrm{L}$ gDNA in a total volume of $25 \mu \mathrm{L}$ per reaction with an ABI Prism 7700 sequence detection system (Applied Biosystems) and the TaqMan Fast Universal PCR Master Mix (Applied Biosystems). The PCR products were analyzed by gel electrophoresis in a 3\% agarose gel stained with ethidium bromide, and the bands were visualized using the ChemiGenius 2 Bio Imaging System (Syngene, Cambridge, UK).

The qPCR reactions were performed as described [11] from gDNA extracted from tissues (diluted $1: 10$ ). The thermocycling conditions consisted of initial denaturation at $95^{\circ} \mathrm{C}$ for $20 \mathrm{sec}$ followed by 45 cycles of $95^{\circ} \mathrm{C}$ for $3 \mathrm{sec}$ and $60^{\circ} \mathrm{C}$ for $45 \mathrm{sec}$. The gDNA was amplified and quantified using a Rotor-Gene 6000 real-time rotary analyzer (Corbett, Mortlake, VIC, Australia) and an ABI Prism 7700 sequence detection system (Applied Biosystems).

2.5. Production of a Standard for Absolute $f A L B$ Quantification. Cat gDNA was used to generate an FALB standard template for absolute quantification. A $150 \mathrm{bp}$ sequence, consisting of the fALB TaqMan sequence, was amplified from $2 \mu \mathrm{L}$ target gDNA using the fALB primers (Table 1). The PCR 
TABLE 1: Details of the TaqMan real-time PCR assays.

\begin{tabular}{|c|c|c|c|c|}
\hline Gene & Oligo & Sequence $\left(5^{\prime} \rightarrow 3^{\prime}\right)$ & Amplicon size (bp) & Final conc. (nM) \\
\hline \multirow{3}{*}{$\mathrm{GAPDH}^{1}$} & Forward & GCCATCAATGACCCCTTCAT & \multirow{3}{*}{82} & 480 \\
\hline & Reverse & GCCGTGGAATTTGCCGT & & 480 \\
\hline & Probe & CTCAACTACATGGTCTACATGTTCCAGTATGATTCCA ${ }^{2}$ & & 160 \\
\hline \multirow{3}{*}{$\mathrm{ALB}^{3}$} & Forward & GATGGCTGATTGCTGTGAGA & \multirow{3}{*}{150} & 500 \\
\hline & Reverse & CCCAGGAACCTCTGTTCATT & & 500 \\
\hline & Probe & ATCCCGGCTTCGGTCAGCTG ${ }^{2,4}$ & & 200 \\
\hline
\end{tabular}

${ }^{1}[10] ;{ }^{2} 5^{\prime}$ FAM $/ 3^{\prime}$ TAMRA; ${ }^{3}$ present study; ${ }^{4}$ HPLC purification.

reaction contained 2.5 units Taq DNA polymerase (Sigma), final concentrations of $250 \mathrm{nM}$ of each primer, $200 \mu \mathrm{M}$ dNTPs (Sigma), $2 \mu \mathrm{L} 10 \times$ PCR buffer (Sigma), and $2 \mu \mathrm{L}$ of template in a final volume of $20 \mu \mathrm{L}$. The amplification was performed using a Biometra TPersonal thermal cycler (Biolabo, Châtel-St-Denis, Switzerland). The cycling conditions consisted of an initial denaturation at $94^{\circ} \mathrm{C}$ for $2 \mathrm{~min}$ followed by 40 cycles of $94^{\circ} \mathrm{C}$ for $30 \mathrm{sec}, 55^{\circ} \mathrm{C}$ for $30 \mathrm{sec}$, and $72^{\circ} \mathrm{C}$ for $15 \mathrm{sec}$, with a final extension at $72^{\circ} \mathrm{C}$ for $2 \mathrm{~min}$. The resulting amplicon was gel-purified and cloned into the TOPO TA cloning vector (Invitrogen). The inserts in selected clones were verified by sequencing using an ABI PRISM 310 genetic analyzer (Applied Biosystems), as described [26]. The fALB reference plasmid was linearized by restriction digestion using the enzyme SalI (Roche, Rotkreuz, Switzerland) and gel-purified (Gen Elute PCR Clean-Up Kit, Sigma-Aldrich), and the copy number was determined spectrophotometrically (GeneQuant, Pharmacia Biotech) and by agarose gel electrophoresis (ChemiGenius 2 Bio Imaging System). Tenfold serial dilutions of the standard templates in $30 \mu \mathrm{g} / \mathrm{mL}$ carrier salmon sperm DNA (Invitrogen) were aliquoted and frozen at $-20^{\circ} \mathrm{C}$, as described [27].

2.6. Efficiency, Analytical Sensitivity, Linear Range, and Precision of the $F A L B$ qPCR Assay. The newly designed fALB qPCR assay was evaluated according to the MIQE guidelines [28] using the Rotor-Gene 6000 real-time rotary analyzer (Corbett). The efficiency of the assay was calculated as previously described [29], using the following equation: $E=$ $\left(10^{(-1 / \text { slope })}\right)-1$. The analytical sensitivity of the system was defined by an endpoint dilution experiment using the tenfold serially diluted standard template and ten replicates per dilution, as described [27]. The linear range of amplification of the fALB qPCR assay was determined by ten-fold serial dilution of the linearized standard template. For the precision analysis, a dilution of a standard template containing $10^{5}$ copies of fALB per reaction was chosen and assayed 10 and 13 times for the intrarun and interrun precision, respectively, and the mean value, standard deviation, and coefficients of variation were calculated.

2.7. Nucleotide Sequence Accession Numbers. The sequence of the partial GAPDH pseudogene of the cat B8 sample (clone 14) was submitted to GenBank under accession number JX523658.

\section{Results}

3.1. Investigation of the GAPDH Pseudogene Sequence. An approach combining sequencing and bioinformatics was chosen to investigate the presence of potential GAPDH pseudogenes in the domestic cat genome. The potential target sequence of the GAPDH qPCR assay was amplified and cloned, and 15 clones were sequenced: 10 from cat B8 and 5 from cat 15 . The sequencing of these 15 clones yielded 11 distinct sequences similar to that of the feline GAPDH mRNA (GenBank: NM_001009307). Eight different sequences were found within the target region of the primers and/or the hydrolysis probe of the GAPDH TaqMan assay (Figure 1). Overall, the sequence identity ranged from $82 \%$ (cat 15, clone 20: cat 15_20, Figure 1) to 96\% (cat B8, clone 9: cat B8_9) in comparison to the reference sequence (GenBank: NM_001009307).

Sequence and gene organization information were retrieved from the Genome Annotation Resource Fields Felis catus v12.2 website (GARFIELD) http://lgd.abcc.ncifcrf.gov/ [30], and five sequences termed "similar to GAPDH" were found in the feline genome. One of these sequences on chromosome F2 exhibited 100\% similarity to clone 14 of cat B8 (cat B8_14, Figure 1). In addition, the GAPDH assay sequence was searched using BLAST against the "AANG WGS Contigs" database containing the Felis catus wholegenome shotgun sequencing project (Felis catus-6.2; 14× coverage; [GenBank: AANG02000000]). Over 60 different contigs containing sequence similar to the GAPDH assay sequence were detected. The sequence with the best fit had three mismatches with the sequence of the GAPDH assay: two mismatches with the forward primer and one mismatch with the reverse primer.

3.2. Efficiency, Analytical Sensitivity, Linear Range, and Precision of the $f A L B$ qPCR Assay. In order to confirm the absence of pseudogenes for feline ALB, a bioinformatics approach was chosen. Sequence and gene organization information was retrieved from the GARFIELD website [30] and the second draft assembly, Felis catus-6.2 (GenBank: AANG02000000). Only one sequence termed "albumin" indicative of the albumin gene, was detected; it is located on chromosome B1.

Subsequently, a fALB real-time PCR assay was designed to amplify a sequence located within the exon IV of the feline albumin. The amplification of feline gDNA using the newly designed primers yielded PCR products with the expected 

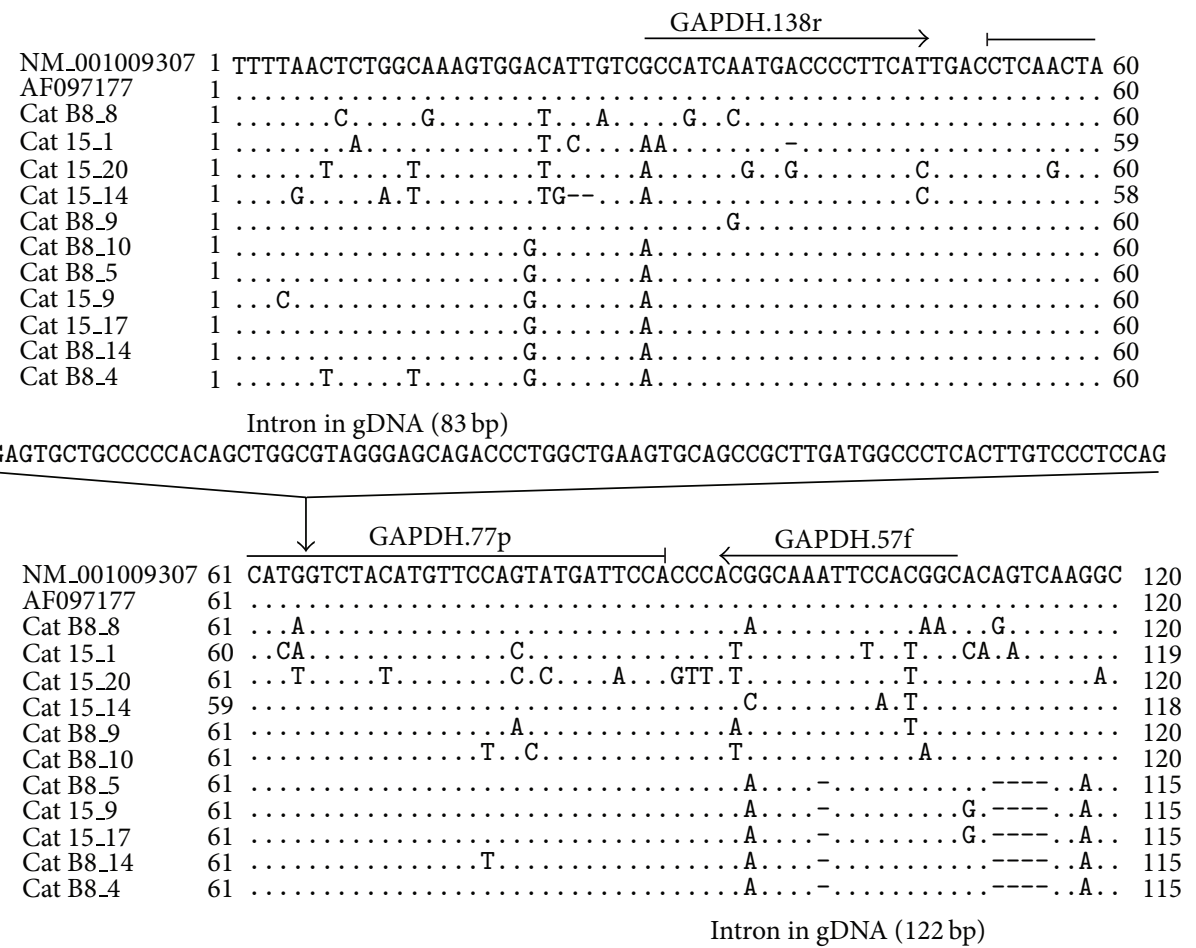

GTAAGTGTAGAAGACGGAACAGGGTGGAATTTGCTTTGAGGGAGTTACTAGGATGGGCTGACAACCTTGGGTGGTACATGGTA CCCCATGTCCCCGAGCTTTCGACTTGTCTCCCTTTATAG

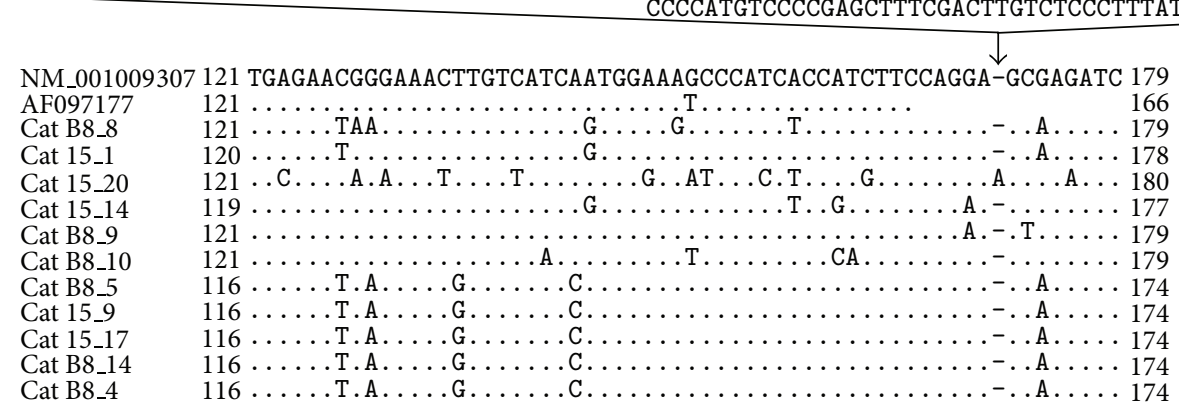

NM_001009307 180 CCGCCAACATCAAATGGG197

\begin{tabular}{|c|c|c|}
\hline AF097177 & 166 & \\
\hline Cat B8_8 & $180 \ldots$. . . . . . . A. 197 & \\
\hline Cat 15_1 & $179 \cdots \ldots \ldots \ldots \ldots \ldots \ldots$ & \\
\hline Cat $15 \_20$ & $181 \cdot T \ldots G \ldots \ldots \ldots \ldots 198$ & $\downarrow$ Splice sites \\
\hline Cat 15_14 & 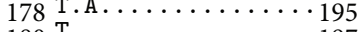 & \\
\hline Cat B8_9 & 180 I $\ldots \ldots \ldots \ldots \ldots \ldots$ & \\
\hline Cat B8_10 & $180 \cdots \cdots \cdots \cdots \cdots \cdots$ & \\
\hline Cat B8_5 & $175 \cdots \cdots \cdots \cdots \cdots \cdots \cdots \cdots$ & \\
\hline Cat 15_9 & $175 \cdots \cdots \cdots \cdots \cdots \cdots \cdots \cdots$ & \\
\hline Cat 15_17 & $175 \cdots \cdots \cdots \cdots \cdots \cdots \cdots \cdots \cdots$ & \\
\hline Cat B8_14 & $175 \cdots \cdots \cdots \cdots \cdots \cdots \cdots$ & \\
\hline Cat B8_4 & $175 \cdots \cdots \cdots \cdots \cdots \cdots \cdots \cdots \cdots$ & \\
\hline
\end{tabular}

FIgURE 1: Comparison of feline GAPDH and pseudo-GAPDH nucleotide sequences. A comparison of the nucleotide sequences of feline GAPDH mRNA (GenBank: NM_001009307, position 60 to 256, and GenBank: AF097177, position 63 to 228) and gDNA sequences similar to GAPDH retrieved from cat B8 and cat 15. The number listed after the animal identifier (B8 and 15) represents the clone identity. Sequences found in multiple clones are shown only once. Nucleotides that differ from those in the two reference strains are indicated. Primer sequences for the qPCR reported previously [10] were located at positions 29 to 48 and 109 to 93 , and the hydrolysis probe spans positions 53 to 89 , as indicated. Arrows point to the splicing sites and the intron sequence from the gDNA (Ensembl: ENSFCAG00000006874) is depicted (Intron in gDNA). The exon sequences of the GAPDH gDNA (Ensembl: ENSFCAG00000006874) on which the GAPDH assay is located (exon 2 and 3) are $100 \%$ identical to the GAPDH mRNA sequence depicted in this figure (GenBank: NM_001009307). 
size of $150 \mathrm{bp}$, as determined by agarose gel electrophoresis. The primers were then used in combination with the newly designed probe, and the amplification efficiency of the fALB qPCR was determined to be $99.5 \%$ to $100 \%$ using 10 -fold serial dilutions of the standard template (Supplementary Material available online at http://dx.doi.org/10.1155/2013/587680 and data not shown). The highest dilution that still resulted in a positive signal in the GPCR assay contained an average of 1 copy of the standard in a $5 \mu \mathrm{L}$ reaction; in an endpoint dilution experiment, 6 of the 10 replicates of this dilution were positive. The fALB qPCR assay was linear over eight orders of magnitude, from $10^{1}$ to $10^{8}$ copies (Supplementary Material). The qPCR assay displayed a good precision; the coefficient of variation for the absolute number using $10^{5}$ copies/reaction was $5.51 \%$ for the intrarun precision analysis and $6.39 \%$ for the interrun analysis.

\section{Discussion}

In this report, we describe the detection of several GAPDHlike sequences that are characteristic for processed GAPDH pseudogenes in the domestic cat genome. Based on the assumption that there is only one copy of the GAPDH pseudogene in the domestic cat genome [14], the GAPDH qPCR assay that was previously designed to amplify GAPDH mRNA/complementary DNA (cDNA) [10] was regularly used to determine the cell number of input gDNA. However, in our experience, the analysis of gDNA samples resulted in a lower amplification efficiency compared to cDNA (unpublished observations); we hypothesized that this might occur due to mismatches between the primers and/or hydrolysis probe and the gDNA sample. Thus, we performed a sequence analysis of the binding region of the primers and the hydrolysis probe of the GAPDH assay. The sequencing of 15 different clones comprising the GAPDH assay sequence revealed 11 different GAPDH-like sequences; however, none exhibited $100 \%$ similarity to the GAPDH mRNA sequence (GenBank: NM_001009307). It is possible that there are additional GAPDH pseudogenes present in the feline genome that were not detected in the present study because the sequencing was restricted to 15 clones. The use of an alternative sequencing technique (deep-sequencing rather than cloning followed by Sanger dideoxy sequencing) may provide a broader picture of the GAPDH-like sequences present in the cat genome. Furthermore, the primer binding sites chosen within the GAPDH sequence may have additionally restricted the number of recognized GAPDH pseudogenes. Thus, the number of GAPDH pseudogenes or GAPDH-like sequences in the feline genome may have been underestimated in our study. However, our sequencing results readily demonstrate that more than one feline GAPDH pseudogene is present in the genomic DNA of feline cells, and the GAPDH pseudogene sequences differed from the GAPDH mRNA sequence to some extent.

This finding was supported by the sequence information retrieved from the GARFIELD website [30] and the second draft assembly, Felis catus-6.2 (GenBank: AANG02000000), in which no sequence with $100 \%$ similarity to the GAPDH mRNA sequence was found, but a multitude of closely related sequences were present. Moreover, our data are in agreement with studies investigating GAPDH pseudogenes in other species. In humans, between 56 and 62 GAPDH pseudogenes have been detected $[15,17]$. Of note, the copy number of pseudogenes may vary within a population, as has been documented for the ATP-binding cassette transporter pseudogene within the Chinese population [31]. The number of recognized processed GAPDH pseudogenes may be as high as 120 in dogs and is over 300 in murine rodents [15-17]. For glycolytic genes, including GAPDH, it has been shown that there is a positive correlation between the level of gene expression and the abundance of processed pseudogenes in mice; GAPDH was found to be particularly highly expressed and to generate the highest number of pseudogenes among all glycolytic genes [17]. The authors explained the overabundance of GAPDH by the fact that GAPDH has many additional functions other than those related to glycolysis [17].

An alternative assay for the quantification of gDNA and cell number in feline samples was designed, validated, and implemented. In other species, different genes have been used for the quantification of cells, including the chemokine receptor CCR5 [32] and ALB [9]. ALB is known to be a single-copy gene in other species, such as humans and rhesus macaques, and has been used in both species as an internal control gene $[8,9,24,25]$. According to the GARFIELD website [30], ALB is also present as a single-copy gene in the feline genome. ALB has been used for the normalization of retroviral provirus loads $[24,25,33,34]$. We chose to develop a feline albumin assay because one of our group's main scientific interests is in retroviral infections. The newly implemented fALB assay was shown to be highly sensitive and efficient for gDNA, with a wide range of linearity. Our database searches resulted in only one hit termed "ALB." Thus, to the best of our knowledge, fALB is a single-copy number gene.

\section{Conclusions}

This study investigated the number of GAPDH pseudogenes in the domestic cat. Our results indicate that several closely related GAPDH-like sequences are indeed present in the cat genome. The GAPDH assay may still be used for quality control to test for the integrity of gDNA and the absence of PCR inhibitors. However, it appears to be a suboptimal choice for the quantification of gDNA equivalents. A newly designed assay using the $\mathrm{AALB}$ reference gene for the normalization of gDNA was validated and implemented. We recommend using this highly sensitive fALB qPCR assay for the normalization of input genomic DNA equivalents in future studies.

\section{Abbreviations}

qPCR: Quantitative real-time PCR

gDNA: Genomic DNA

GAPDH: Glyceraldehyde-3-phosphate dehydrogenase

fALB: $\quad$ Feline albumin

mRNA: Messenger RNA 
cDNA: Complementary DNA

CT: Cycle threshold.

\section{Conflict of Interests}

The authors declare that they have no conflict of interests.

\section{Authors' Contribution}

A. Katrin Helfer-Hungerbuehler performed the experiments, analyzed the data, and drafted the paper. Stefan Widmer performed the sequencing experiments as a partial fulfillment of the requirement of a term paper. RHL conceived and supervised the follow-up study and edited the paper.

\section{Acknowledgments}

The authors would particularly like to thank Dr. V. Cattori and Dr. M. L. Meli for excellent assistance and helpful discussions. They are grateful to the animal caretakers for expert technical aid with the cats and to the technicians and doctoral students of the Clinical Laboratory, particularly T. Meili Prodan and B. Weibel, and the Institute for Veterinary Pathology, Vetsuisse Faculty, for their excellent laboratory assistance. The molecular biology work was performed using the logistics of the Center for Clinical Studies, Vetsuisse Faculty, University of Zurich. This study was financially supported by a Research Grant from the Swiss National Science Foundation (310030_135586).

\section{References}

[1] M. Kubista, J. M. Andrade, M. Bengtsson et al., "The real-time polymerase chain reaction," Molecular Aspects of Medicine, vol. 27, no. 2-3, pp. 95-125, 2006.

[2] S. A. Bustin, "Absolute quantification of mRNA using real-time reverse transcription polymerase chain reaction assays," Journal of Molecular Endocrinology, vol. 25, no. 2, pp. 169-193, 2000.

[3] P. S. Bernard and C. T. Wittwer, "Real-time PCR technology for cancer diagnostics," Clinical Chemistry, vol. 48, no. 8, pp. 11781185, 2002.

[4] I. M. Mackay, K. E. Arden, and A. Nitsche, "Real-time PCR in virology," Nucleic Acids Research, vol. 30, no. 6, pp. 1292-1305, 2002.

[5] I. M. Mackay, "Real-time PCR in the microbiology laboratory," Clinical Microbiology and Infection, vol. 10, no. 3, pp. 190-212, 2004.

[6] S. A. Bustin and R. Mueller, "Real-time reverse transcription PCR (qRT-PCR) and its potential use in clinical diagnosis," Clinical Science, vol. 109, no. 4, pp. 365-379, 2005.

[7] L. I. Moreno, C. M. Tate, E. L. Knott et al., "Determination of an effective housekeeping gene for the quantification of mRNA for forensic applications," Journal of Forensic Sciences, vol. 57, no. 4, pp. 1051-1058, 2012.

[8] I. Laurendeau, M. Bahuau, N. Vodovar et al., "TaqMan PCRbased gene dosage assay for predictive testing in individuals from a cancer family with INK4 locus haploinsufficiency," Clinical Chemistry, vol. 45, no. 7, pp. 982-986, 1999.
[9] I. Bieche, M. H. Champème, D. Vidaud, R. Lidereau, and M. Vidaud, "Novel approach to quantitative polymerase chain reaction using real-time detection: application to the detection of gene amplification in breast cancer," International Journal of Cancer, vol. 78, no. 5, pp. 661-666, 1998.

[10] C. M. Leutenegger, C. N. Mislin, B. Sigrist, M. U. Ehrengruber, R. Hofmann-Lehmann, and H. Lutz, "Quantitative real-time PCR for the measurement of feline cytokine mRNA," Veterinary Immunology and Immunopathology, vol. 71, no. 3-4, pp. 291-305, 1999.

[11] Y. Kessler, A. K. Helfer-Hungerbuehler, V. Cattori et al., "Quantitative TaqMan ${ }^{\circledR}$ real-time PCR assays for gene expression normalisation in feline tissues," BMC Molecular Biology, vol. 10, article no. 106, 2009.

[12] G. A. Wolf-Jackel, V. Cattori, C. P. Geret et al., "Quantification of the humoral immune response and hemoplasma blood and tissue loads in cats coinfected with "Candidatus Mycoplasma haemominutum" and feline leukemia virus," Microbial Pathogenesis, vol. 53, no. 2, pp. 74-80, 2012.

[13] M. Novacco, F. S. Boretti, G. A. Wolf-Jackel et al., "Chronic, "Candidatus Mycoplasma turicensis" Infection," Veterinary Research, vol. 42, no. 1, article 59, 2011.

[14] S. Molia, B. B. Chomel, R. W. Kasten et al., "Prevalence of Bartonella infection in wild African lions (Panthera leo) and cheetahs (Acinonyx jubatus)," Veterinary Microbiology, vol. 100, no. 1-2, pp. 31-41, 2004.

[15] Y. J. Liu, D. Zheng, S. Balasubramanian et al., "Comprehensive analysis of the pseudogenes of glycolytic enzymes in vertebrates: the anomalously high number of GAPDH pseudogenes highlights a recent burst of retrotrans-positional activity," $B M C$ Genomics, vol. 10, article 480, 2009.

[16] S. Riad-El Sabrouty, J. M. Blanchard, L. Marty, P. Jeanteur, and M. Piechaczyk, "The Muridae glyceraldehyde-3-phosphate dehydrogenase family," Journal of Molecular Evolution, vol. 29, no. 3, pp. 212-222, 1989.

[17] L. McDonell and G. Drouin, "The abundance of processed pseudogenes derived from glycolytic genes is correlated with their expression level," Genome, vol. 55, no. 2, pp. 147-151, 2012.

[18] Z. Zhang, N. Carriero, and M. Gerstein, "Comparative analysis of processed pseudogenes in the mouse and human genomes," Trends in Genetics, vol. 20, no. 2, pp. 62-67, 2004.

[19] P. Ruud, O. Fodstad, and E. Hovig, "Identification of a novel cytokeratin 19 pseudogene that may interfere with reverse transcriptase-polymerase chain reaction assays used to detect micrometastatic tumor cells," International Journal of Cancer, vol. 80, no. 1, pp. 119-125, 1999.

[20] B. Garbay, E. Boue-Grabot, and M. Garret, "Processed pseudogenes interfere with reverse transcriptase-polymerase chain reaction controls," Analytical Biochemistry, vol. 237, no. 1, pp. 157-159, 1996.

[21] C. P. Geret, B. Riond, V. Cattori, M. L. Meli, R. HofmannLehmann, and H. Lutz, "Housing and care of laboratory cats: from requirements to practice," Schweizer Archiv fur Tierheilkunde, vol. 153, no. 4, pp. 157-164, 2011.

[22] A. K. Helfer-Hungerbuehler, V. Cattori, F. S. Boretti et al., "Dominance of highly divergent feline leukemia virus A progeny variants in a cat with recurrent viremia and fatal lymphoma," Retrovirology, vol. 7, no. 1, article 14, 2010.

[23] M. Kullberg, M. A. Nilsson, U. Arnason, E. H. Harley, and A. Janke, "Housekeeping genes for phylogenetic analysis of eutherian relationships," Molecular Biology and Evolution, vol. 23, no. 8, pp. 1493-1503, 2006. 
[24] A. Dehée, R. Césaire, N. Désiré et al., "Quantitation of HTLVI proviral load by a TaqMan real-time PCR assay," Journal of Virological Methods, vol. 102, no. 1-2, pp. 37-51, 2002.

[25] H. K. Chung, T. Unangst, J. Treece, D. Weiss, and P. Markham, "Development of real-time PCR assays for quantitation of simian betaretrovirus serotype-1, $-2,-3$, and -5 viral DNA in Asian monkeys," Journal of Virological Methods, vol. 152, no. 1-2, pp. 91-97, 2008.

[26] B. Willi, F. S. Boretti, V. Cattori et al., "Identification, molecular characterization, and experimental transmission of a new hemoplasma isolate from a cat with hemolytic anemia in Switzerland," Journal of Clinical Microbiology, vol. 43, no. 6, pp. 2581-2585, 2005.

[27] V. Cattori and R. Hofmann-Lehmann, "Absolute quantitation of feline leukemia virus proviral DNA and viral RNA loads by TaqMan real-time PCR and RT-PCR," Methods in Molecular Biology, vol. 429, pp. 73-87, 2008.

[28] S. A. Bustin, V. Benes, J. A. Garson et al., “The MIQE guidelines: minimum information for publication of quantitative real-time PCR experiments," Clinical Chemistry, vol. 55, no. 4, pp. 611-622, 2009.

[29] D. Klein, P. Janda, R. Steinborn, M. Müller, B. Salmons, and W. H. Günzburg, "Proviral load determination of different feline immunodeficiency virus isolates using real-time polymerase chain reaction: influence of mismatches on quantification," Electrophoresis, vol. 20, no. 2, pp. 291-299, 1999.

[30] J. U. Pontius and S. J. O’Brien, "Genome annotation resource fields-GARFIELD: a genome browser for Felis catus," Journal of Heredity, vol. 98, no. 5, pp. 386-389, 2007.

[31] M. K. Kringen, C. Stormo, R. M. Grimholt, J. P. Berg, and A. P. Piehler, "Copy number variations of the ATP-binding cassette transporter ABCC6 gene and its pseudogenes," BMC Research Notes, vol. 5, no. 1, article 425, 2012.

[32] J. A. Thomas, T. D. Gagliardi, W. G. Alvord, M. Lubomirski, W. J. Bosche, and R. J. Gorelick, "Human immunodeficiency virus type 1 nucleocapsid zinc-finger mutations cause defects in reverse transcription and integration," Virology, vol. 353, no. 1, pp. 41-51, 2006.

[33] N. Désiré, A. Dehée, V. Schneider et al., "Quantification of human immunodeficiency virus type 1 proviral load by a TaqMan real-time PCR assay," Journal of Clinical Microbiology, vol. 39, no. 4, pp. 1303-1310, 2001.

[34] A. Waters, A. L. A. Oliveira, S. Coughlan et al., "Multiplex realtime PCR for the detection and quantitation of HTLV-1 and HTLV-2 proviral load: addressing the issue of indeterminate HTLV results," Journal of Clinical Virology, vol. 52, no. 1, pp. 3844, 2011. 

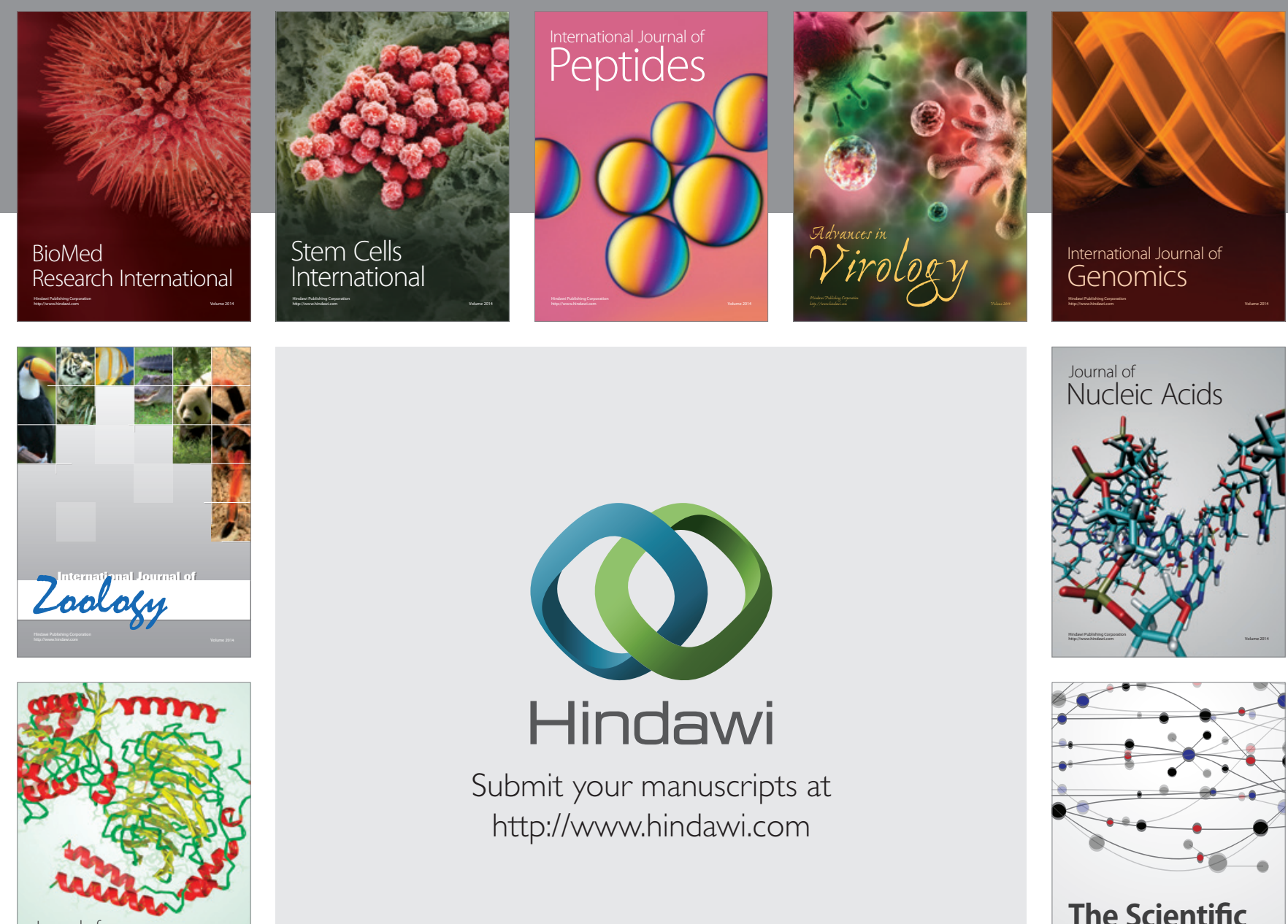

Submit your manuscripts at

http://www.hindawi.com

Journal of
Signal Transduction
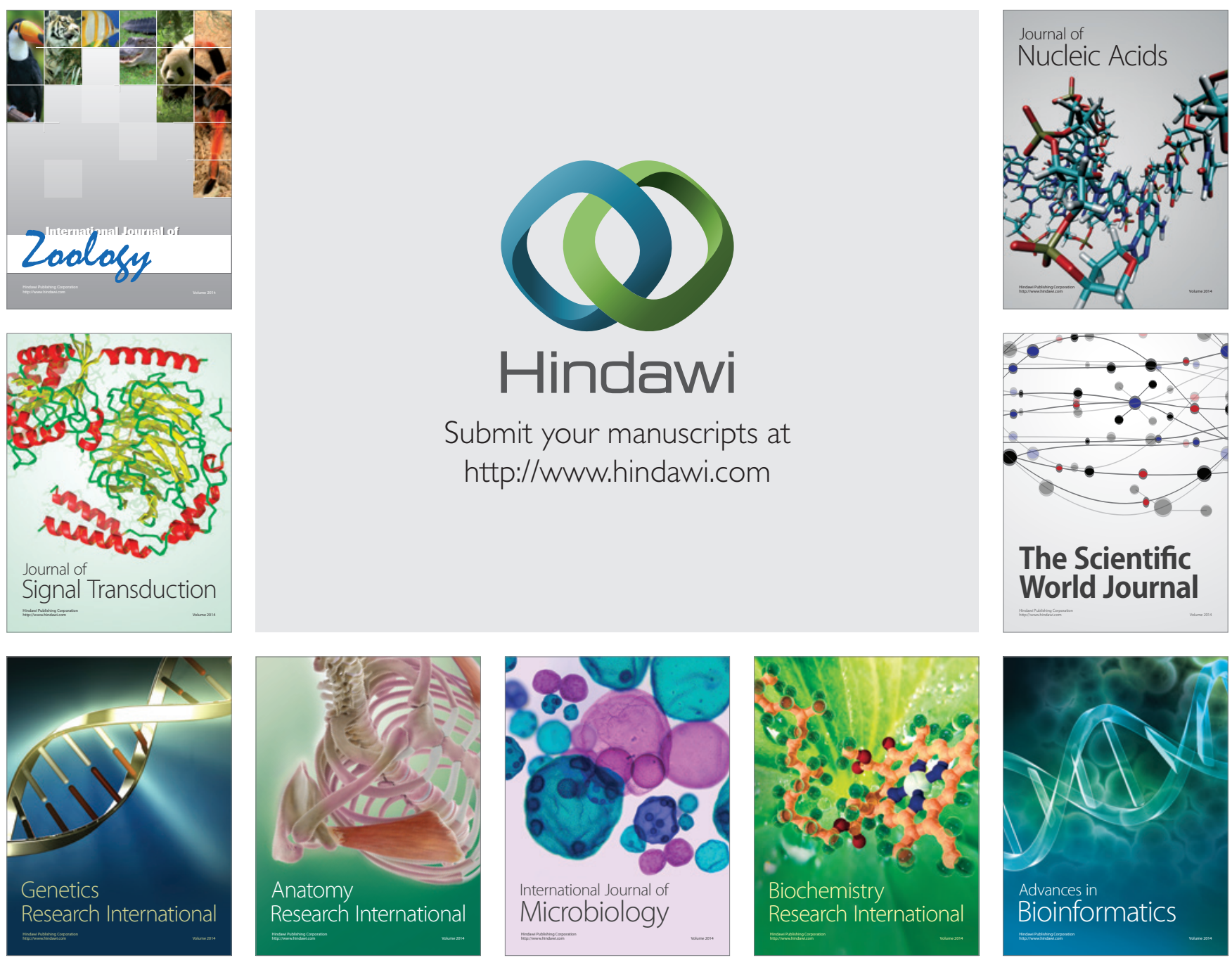

The Scientific World Journal
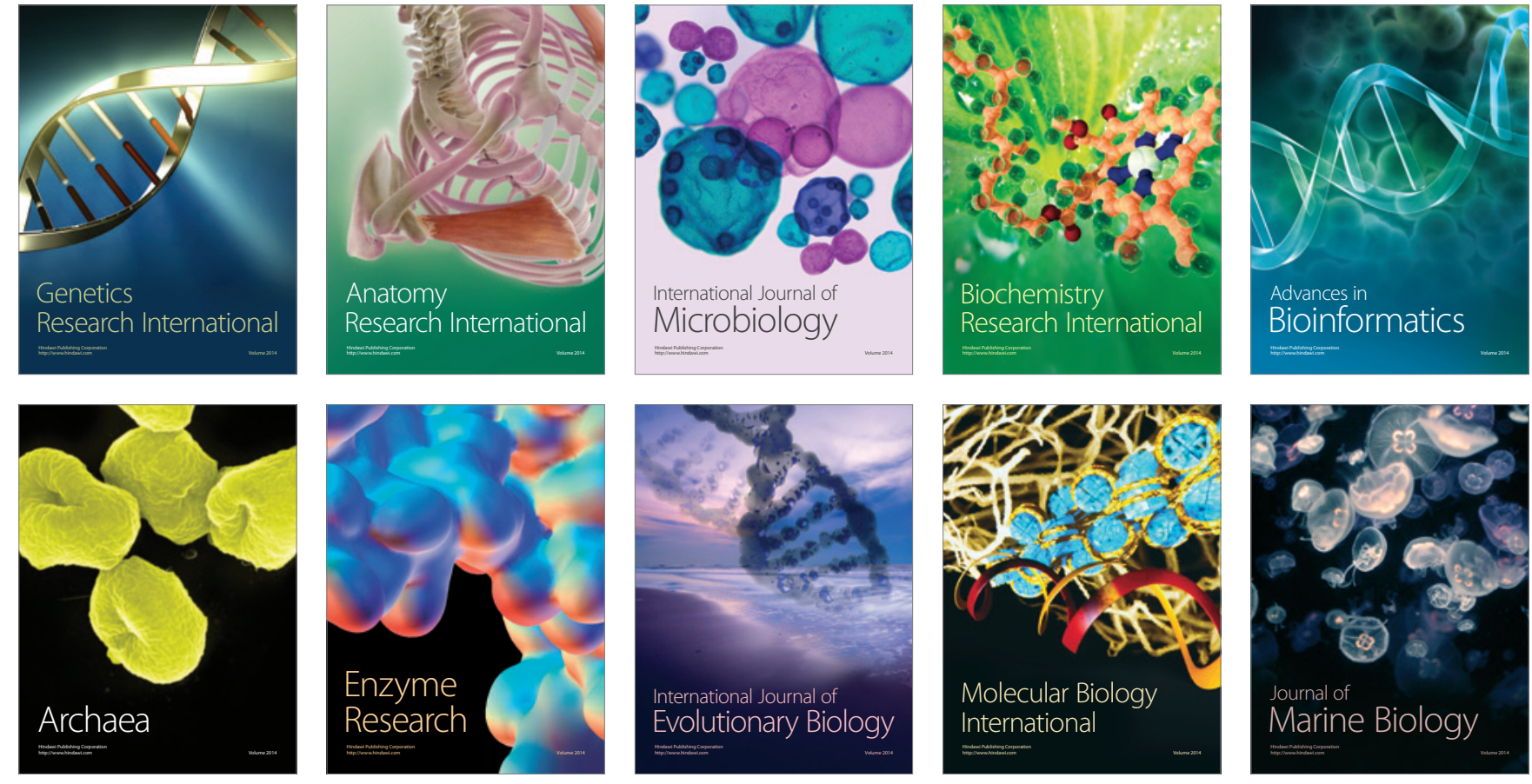